\title{
Estrogenic Compounds Compatible with a Conditional Gene Expression System for the Phytopathogenic Fungus Fusarium graminearum
}

\author{
Jungkwan Lee ${ }^{1 *}$, Hokyoung Son ${ }^{2}$ and Yin-Won Lee ${ }^{2}$ \\ ${ }^{1}$ Department of Applied Biology, Dong-A University, Busan 604-714, Korea \\ ${ }^{2}$ Department of Agricultural Biotechnology and Center for Fungal Pathogenesis, Seoul National University, Seoul 151-921, \\ Korea
}

(Received on September 17, 2011; Accepted on October 10, 2011)

The ascomycete fungus Fusarium graminearum is an important plant pathogen responsible for Fusarium head blight in small grains and ear rot on maize. This fungus also produces the estrogenic metabolite, zearalenone (ZEA) that causes estrogenic disorders in humans and animals. Previously, we developed a conditional gene expression system for this fungus using a ZEA-inducible promoter (Pzear). In the present study, four other estrogenic compounds, including $\beta$-estradiol, estriol, estrone, and secoisolariciresinol, were screened as possible substitutes for ZEA in this system. Among them, $\beta$ estradiol was able to successfully induce the expression of a gene controlled by Pzear, while estrone was only able to partially induce its expression but the other two compounds were not effective. In combination, these results demonstrate that $\beta$-estradiol can replace ZEA in this conditional gene expression system, thereby eliminating the need to use the more expensive reagent, ZEA, and facilitating high-throughput functional analyses of $\boldsymbol{F}$. graminearum in future studies.

Keywords : conditional gene expression, estrogens, estradiol, Gibberella zeae, zearalenone

The plant pathogenic fungus Fusarium graminearum (teleomorph: Gibberella zeae) is responsible for causing Fusarium head blight (FHB) on small grains and ear rot on maize (Leslie and Summerell, 2006). Unfortunately, both of these diseases have recently been reported in the rice and maize fields of Korea (Lee et al., 2009, 2010c). In addition to direct yield losses as a result of disease on heads, this fungus also produces various mycotoxins, including trichothecenes and zearalenone (ZEA) (Desjardins, 2006). Trichothecenes inhibit eukaryotic protein biosynthesis, which leads to food refusal, diarrhea, and contact dermatitis in animals, while trichothecenes represent virulence factors

\footnotetext{
*Corresponding author.

Phone) +82-51-200-7554, FAX) +82-51-200-7505

E-mail) jungle@dau.ac.kr
}

in plants. ZEA [6-(10-hydroxy-6-oxo-trans-1-undecenyl)$\beta$-resorcyclic acid lactone] is a polyketide metabolite and is responsible for estrogenic disorders in rats and swine due to the similarity of its structure with animal estrogens (Desjardins, 2006).

The ZEA biosynthesis gene cluster of $F$. graminearum includes four genes, a reducing polyketide synthase (PKS4), non-reducing polyketide synthase (PKS13), isoamyl alcohol oxidase (ZEB1), and transcription factor (ZEB2) (Gaffoor and Trail, 2006; Kim et al., 2005; Lysøe et al., 2006). The two PKS genes synthesize a polyketide backbone through the head-to-tail condensation of acetate units, and ZEB1 plays an important role for an oxidation step which converts $\beta$-zearalenol ( $\beta$-ZOL) to ZEA (Kim et al., 2005). ZEB2 contains a putative bZIP DNA-binding domain and is a transcriptional activator of the other three genes in the cluster. Recently, a putative ABC transporter gene, ZRA1, has been identified to be required for ZEA production in this fungus even though its biological function was not elucidated yet (Lee et al., 2011b). These functional studies revealed the ZEA biosynthetic pathway, but biological functions of ZEA in plants and fungi have not yet been characterized.

Since the whole genome sequences of $F$. graminearum were publicly released by the Broad Institute in 2003 (http:/ /www.broadinstitute.org/), many research groups have performed functional studies to identify genes related to pathogenicity (Ding et al., 2009; Seong et al., 2005), sexual and asexual development (Harris, 2005; Min et al., 2010; Lee et al., 2009, 2010a; Son et al., 2011), and secondary metabolite biosynthesis pathways (Gaffoor and Trail, 2006; Kim et al., 2005, 2006; Lysøe et al., 2006). While molecular manipulation tools such as targeted gene deletion and gene over-expression have been well developed, conditional gene expression systems that allow the expression of certain genes under desired conditions have been limited in $F$. graminearum. Currently, the only conditional gene expression system for $F$. graminearum involves a ZEAinducible promoter we previously developed (Lee et al., 
2010b). Despite the high efficiency of this system in studying essential genes, including the selective expression of certain genes, the high cost associated with obtaining ZEA limits large-scale functional studies. Therefore, the goal of this study was to evaluate the capacity for four other estrogenic compounds, each with structures similar to ZEA, to substitute for ZEA in a conditional gene expression system for $F$. graminearum.

Fungal strains, media, and estrogenic compounds. The F. graminearum wild-type strain GZ03639 was provided by Robert L. Bowden (U.S. Department of AgricultureAgricultural Research Service, Manhattan, KS, USA). Negative and positive controls used in this study included the $F$. graminearum methionine auxotroph, $\Delta$ gzmetE (Han et al., 2004), and the Pzear-GzMetE mutant strain with the methionine biosynthesis gene promoter replaced by a ZEAinducible promoter (Pzear) (Lee et al., 2010b), respectively. All strains were maintained on complete medium (CM), while studies of each estrogenic compound were performed in either CM or minimal medium (MM) (Leslie and Summerell, 2006) supplemented with each estrogenic compound. For the long-term storage, all strains were frozen as a conidial suspension in $15 \%$ glycerol at $-70{ }^{\circ} \mathrm{C}$. ZEA, one plant estrogen (secoisolariciresinol), and three animal estrogens ( $\beta$-estradiol, estrone, and estriol) were purchased from Sigma-Aldrich (St. Louis, MO, USA).
Each compound was dissolved in methanol to a final concentration of $10 \mathrm{mM}$.

Screening for the activity of estrogenic compounds. Small agar blocks $\left(1 \mathrm{~mm}^{3}\right)$ from CM cultures were placed on MM without estrogenic compounds and MM supplemented with $30 \mu \mathrm{M}$ of each estrogenic compound and incubated for $3 \mathrm{~d}$ at $25^{\circ} \mathrm{C}$ in the dark. Consistent with the previous report (Lee et al., 2010b), the wild-type strain grew well independent of ZEA, while the $\Delta$ gzmetE and Pzear-GzMetE strains did not grow in MM without ZEA (Fig. 1). The Pzear-GzMetE strain also did not grow on MM supplemented with secoisolariciresinol or estriol. When MM was supplemented with estrone, growth of each of the mutants was partially restored. However, the mycelia growth restoration on the medium was not consistent among strains and each strain did not show radial growth (Fig. 1). This result suggests that the fungal strains do not efficiently take estrone in their cells or estrone is not properly working for the gene expression in the cells. MM supplemented with $\beta$-estradiol completely restored the growth of the mutants to that of the wild-type strain (Fig. 1). In combination, these results suggest that the methionine biosynthesis gene, GzMetE, is induced in the presence of $\beta$ estradiol, and $\beta$-estradiol represents a potential substitute for ZEA in the conditional gene expression system for $F$. graminearum.
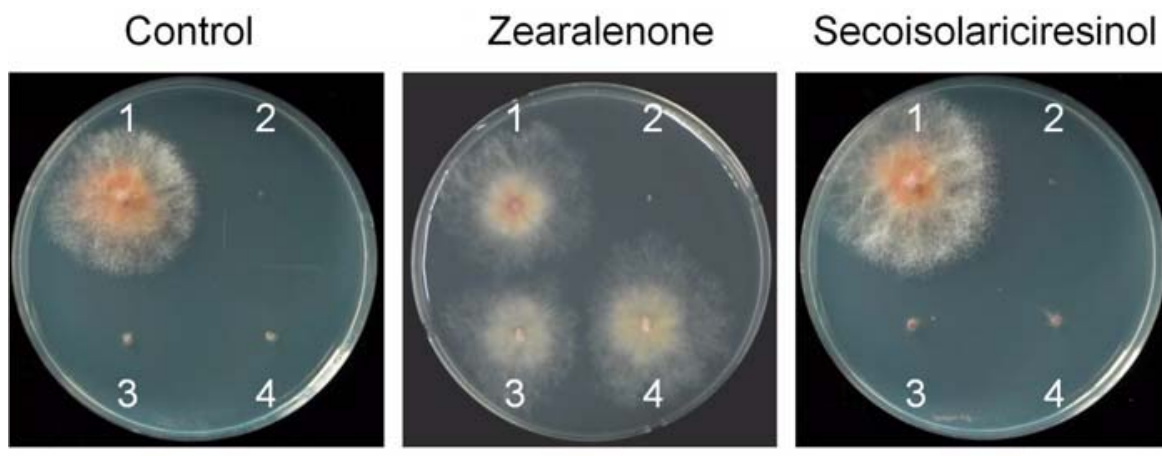

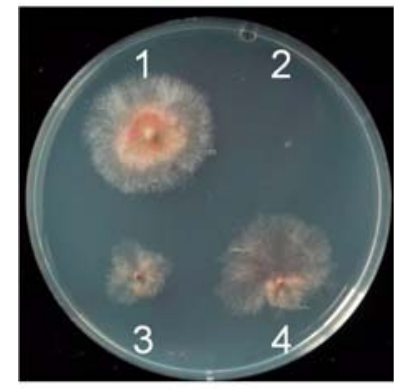

Estrone

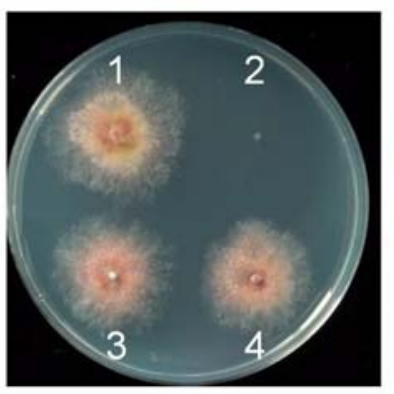

$\beta$-estradiol
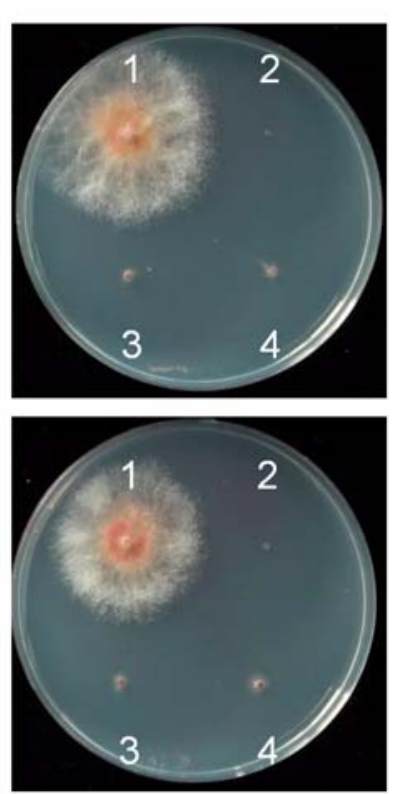

Estriol

Fig. 1. Mycelial growth was monitored on minimal medium (MM) supplemented with $30 \mu \mathrm{M}$ of the estrogenic compounds indicated. Growth of the following F. graminearum strains were observed: 1: wild-type GZ03639; 2: $\Delta g z m e t E ; 3$ and 4: Pzear-GzMetE mutants. Photographs were taken 3 days after inoculation. 
Table 1. Primers used in this study

\begin{tabular}{ll}
\hline \hline Primer & Sequence $\left(5^{\prime}-3^{\prime}\right)$ \\
\hline HYG-F1 & GGCTTGGCTGGAGCTAGTGGAGG \\
zear-r2 & CATGGTTACTTTCGTTCTCTCTGGTC \\
ACS2-5F & TGAAGGAAGCTGGGTTTGTTGATG \\
ACS2-5R pzear & TCCACTAGCTCCAGCCAAGCCGGTCAAGGATATCCCTGCTGAAAGT \\
ACS2-3F pzear & GAGAGAACGAAAGTAACCATGTACCTAATCATGCGACAAAACTTACC \\
ACS2-3R pzear & TGGGCAGATCAGGCACAACAAA \\
ACS2-5N & TGGAGGCTGGGCAAAAGACGA \\
ACS2-3N pzear & CAACAGAGTGAACAGCGCCGATAC \\
\hline
\end{tabular}

Replacement of the ACS2 promoter with Pzear in the $A C L 2$ deletion mutant. Previously, it was demonstrated that the deletion of both acetyl-CoA synthase 2 (ACS2) and ATP citrate lyase 2 (ACL2) genes resulted in a lethal phenotype since double deletion mutants were unable to be obtained following repeated transformation trials and outcrossing between single mutants (Lee et al., 2011a). The replacement of the ACS2 promoter with Pzear in the ACL2 deletion mutant resulted in a severe reduction in mycelia growth but its defect was partially recovered with the addition of ZEA to the growth medium (Lee et al., 2011a). In the present study, the same strategy was used to replace the $A C S 2$ promoter with Pzear in the ACL2 deletion mutant ( $\triangle a c l 2 A C S 2 p$ ) (Son et al., 2011), yet $\beta$-estradiol was used instead of ZEA. In brief, the hyg-pzear fragment that carries a hygromycin-resistant gene fused with Pzear was amplified from the Pzear-GzmetE strain (Lee et al., 2010b) using HYG-F1 and zear-r2 primers. Amplifications of the $5^{\prime}$ flanking region and a partial sequence of the $A C S 2$ gene were also performed using GZ03639 and primers, ACS25F/ACS2-5R pzear and ACS2-3F pzear/ACS2-3R pzear, respectively (Table 1). The three fragments obtained were then fused using the double-joint PCR method (Yu et al., 2004), and the final construct was amplified using ACS25N/ACS2-3N pzear primers. Using polyethylene glycol (PEG)-mediated transformation (Kim et al., 2006), the final construct was introduced into protoplasts of the $A C L 2$ deletion mutant and grown on media supplemented with 30 $\mu \mathrm{M} \beta$-estradiol during the regeneration and selection processes.

From one transformation trial, seven transformants were isolated and each transformant was grown in $50 \mathrm{ml}$ of CM supplemented with $30 \mu \mathrm{M} \beta$-estradiol for $3 \mathrm{~d}$ on a rotary shaker (150 rpm). Fungal genomic DNA was then prepared using a cetyl trimethyl ammonium bromide protocol (Leslie and Summerell, 2006). Standard procedures were used for subsequent restriction endonuclease digestions and agarose gel electrophoresis (Sambrook and Russell, 2001). Southern blot hybridizations were also performed at $65^{\circ} \mathrm{C}$ for $18 \mathrm{~h}$ with $100 \mathrm{ng}$ of $5^{\prime}$ flanking region of the ACS2 gene labeled with $\left[\alpha-{ }^{32} \mathrm{P}\right] \mathrm{dCTP}$ by random priming and used as a probe. Positive mutant clones were confirmed by the presence of a $5.9 \mathrm{~kb}$ band, while the wild-type construct migrated as a 3.8 $\mathrm{kb}$ band (Fig. 2A). Desired mutants exhibited severe growth inhibition on CM, yet in the presence of $\beta$-estradiol, their growth was partially restored to resemble that of a mutant containing only a single $A C L 2$ deletion (Fig. 2B).

The results of the present study demonstrate that $\beta$ estradiol can be successfully substituted for ZEA in the conditional gene expression system previously developed for $F$. graminearum (Lee et al., 2010b), thereby providing an alternative to ZEA that is approximately 100 times less expensive. Accordingly large-scale functional studies of essential genes in this fungus may be more accessible. Moreover, $\beta$-estradiol was shown to be applicable to $F$. oxysporum and $F$. verticillioides transformants carrying green fluorescent protein (GFP) fused with a ZEAinducible promoter (Lee et al., 2010b). The level of GFP expression in the strains was dependent on the $\beta$-estradiol concentration (data not shown). In combination, these results suggest that $\beta$-estradiol can be broadly applied to experimental systems involving the genus Fusarium.

The phytoestrogen secoisolariciresinol is a polyphenolic substance to be metabolized into enterodiol and enterolactone by intestinal bacteria when consumed as part of the diet by mammalians (Lampe, 2003). The metabolized compounds bind to estrogen receptors localized in the cytosol and/or nucleus to regulate the expression of estrogen-responsive genes (Wang, 2002). Secoisolariciresionl is one component of wheat gran (Smeds et al., 2007), and we expected that it can be substituted for ZEA if it is metabolized in fungal cells as shown in mammalian cells. Our result, however, showed that it is not working for the expression of GzMetE controlled by Pzear (Fig. 1). Interestingly, the $F$. graminearum wild-type strain showed slightly increased growth rate and aerial mycelia in the MM supplemented with secoisolariciresinol (Fig. 1). We do not know whether secoisolariciresionl is metabolized in the 
A

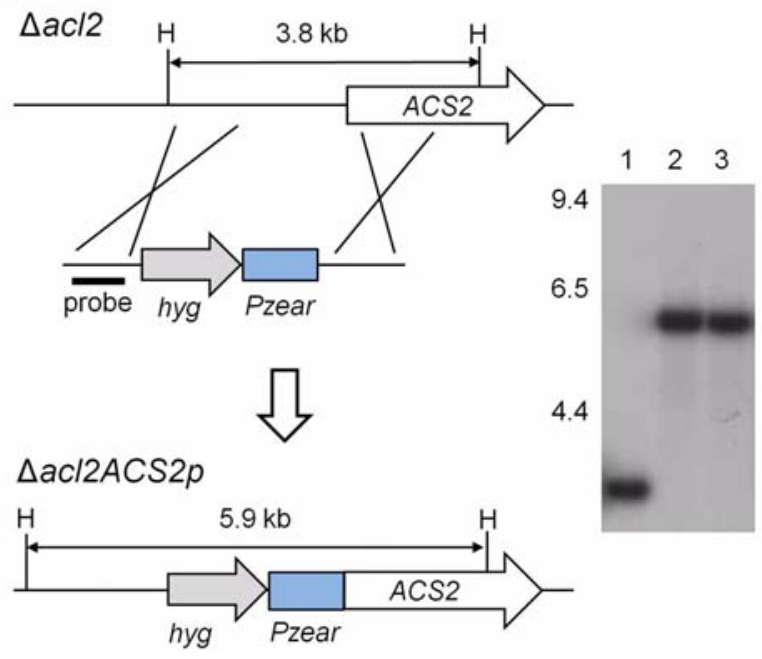

B

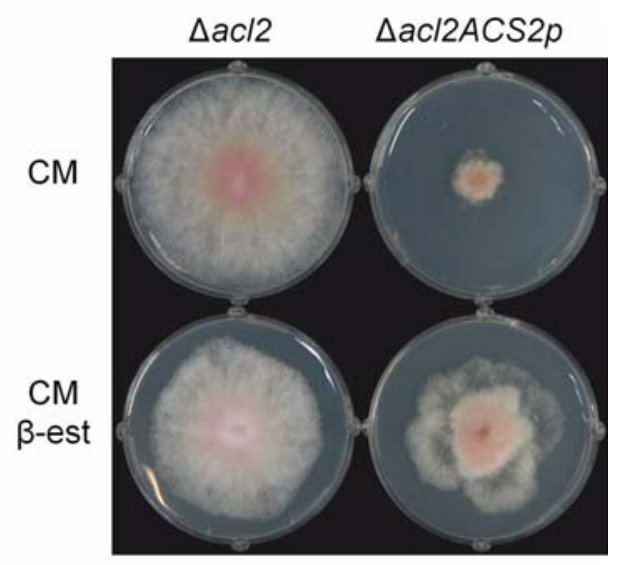

Fig. 2. Characterization of a construct containing the ACS2 gene under the control of a ZEA-inducible promoter (Pzear). (A) An overview of the strategy used to fuse a hyg-Pzear fragment amplified from the Pzear-GzMetE strain with the 5' flanking region and partial sequence of the $A C S 2$ gene to provide a final construct that was introduced into an $A C L 2$ deletion mutant. Southern blot hybridization assays confirmed that the wild-type strain, GZ03639 (lane 1), migrated as a $3.8 \mathrm{~kb}$ fragment, while the positive mutant clones (lanes 2 and 3) migrated as $5.9 \mathrm{~kb}$ bands. (B) Mycelial growth of $\triangle a c l 2$ (an ACL2 deletion mutant) and $\triangle a c l 2 A C S 2 p$ (an ACL2 deletion mutant with the $A C S 2$ gene under the control of Pzear) observed on CM in the absence and presence of $30 \mu \mathrm{M}$ of $\beta$-estradiol ( $\beta$ est).

fungal cells, but our result suggests that the wild-type $F$. graminearum strain uses the compound as a carbon source. This consumption may help $F$. graminearum colonize wheat gran at the early infection stage.

Estrogenic compounds produced by animals readily diffuse across the cell membrane and they are signaling molecules that exert their effects by binding to estrogen receptors. The estrogen-receptor complex interacts with DNA to regulate gene expression (Nussey and Whitehead, 2001). In addition, estrogens have been shown to activate a $\mathrm{G}$ protein-coupled receptor, GPR30, to promote inhibition of oxidative stress-induced apoptosis and cell growth (Kanda and Watanabe, 2003a, b). In studies of ZEA, the capacity for this fungal estrogen to act as a sex hormone in the sexual reproduction in F. graminearum was observed (Wolf and Mirocha, 1973), yet ZEA-non-producing mutants also maintained the ability to produce a significant number of perithecia (Kim et al., 2005). To date, neither receptors for ZEA, nor biological functions of ZEA, have been characterized in a fungal system. However, grains infected by $F$. graminearum are significantly contaminated with ZEA and most $F$. graminearum field strains are able to produce ZEA (Leslie and Summerell, 2006; Lee et al., 2009), suggesting that ZEA has certain biological functions for this fungus. Our previous studies showed that exogenous treatment of ZEA into medium before $F$. graminearum inoculation altered expression levels of several hundred genes even though the treatment did not trigger any pheno- typic changes (Lee et al., 2011a). Therefore, it is significant that we demonstrate that ZEA may mediate similar mechanism(s) as $\beta$-estradiol in regulating gene expression, thereby facilitating future studies to elucidate the biological functions of ZEA in fungal systems.

\section{Acknowledgements}

This work was supported by the National Research Foundation of Korea (NRF) grant funded by the Korean government (MEST) (2011-0000963) and by the Basic Science Research Program through the NRF funded by the Ministry of Education, Science and Technology (20110003021).

\section{References}

Desjardins, A. E. 2006. Fusarium mycotoxins: Chemistry, genetics and biology. APS Press, St. Paul, MN.

Ding, S., Mehrabi, R., Koten, C., Kang, Z., Wei, Y., Seong, K., Kistler, H. C. and Xu, J.-R. 2009. Transducin beta-like gene FTL1 is essential for pathogenesis in Fusarium graminearum. Eukaryot. Cell 8:867-876.

Gaffoor, I. and Trail, F. 2006. Characterization of two polyketide synthase genes involved in zearalenone biosynthesis in Gibberella zeae. Appl. Environ. Microbiol. 72:1793-1799.

Han, Y.-K., Lee, T., Han, K.-H., Yun, S.-H. and Lee, Y.-W. 2004. Functional analysis of the homoserine O-acetyltransferase gene and its identification as a selectable marker in Gibberella 
zeae. Curr. Genet. 46:205-212.

Harris, S. D. 2005. Morphogenesis in germinating Fusarium graminearum macroconidia. Mycologia 97:880-887.

Kanda, N. and Watanabe, S. 2003a. 17 $\beta$-estradiol enhaces the production of nerve growth factor in THP-1-derived macrophages or peripheral blood monocyte-derived macrophages. J. Invest. Dermatol. 121:771-780.

Kanda, N. and Watanabe, S. 2003b. 17 $\beta$-estradiol inhibits oxidative stress-induced apoptosis in keratinocytes by promoting Bcl-2 expression. J. Invest. Dermatol. 121:1500-1509.

Kim, J.-E., Jin, J., Kim, H., Kim, J.-C., Yun, S.-H. and Lee, Y.-W. 2006. GIP2, a putative transcription factor that regulates the aurofusarin biosynthetic gene cluster in Gibberella zeae. Appl. Environ. Microbiol. 72:1645-1652.

Kim, Y.-T., Lee, Y.-R., Jin, J., Han, K.-H., Kim, H., Kim, J.-C., Lee, T., Yun, S.-H. and Lee, Y.-W. 2005. Two different polyketide synthase genes are required for synthesis of zearalenone in Gibberella zeae. Mol. Microbiol. 58:1102-1113.

Lampe, J. W. 2003. Isoflavonid and lignan phytoestrogens as dietary biomarkers. J. Nutr. 133: 956S-964S.

Lee, J., Chang, I.-Y., Kim, H., Yun, S.-H., Leslie, J. F. and Lee, Y.W. 2009. Genetic diversity and fitness of Fusarium graminearum populations from rice in Korea. Appl. Envirion. Microbiol. 75:3289-3295.

Lee, J., Park, C.-J., Kim, J.-C., Kim, J.-E. and Lee, Y.-W. 2010a. Identification and functional characterization of genes involved in the sexual reproduction of the ascomycete fungus Giberella zeae. Biochem. Biophys. Res. Commun. 401:48-52.

Lee, J., Son, H., Lee, S., Park, A. R. and Lee, Y.-W. 2010b. Development of a conditional gene expression system using a zearalenone-inducible promoter for the ascomycete fungus Gibberella zeae. Appl. Environ. Microbiol. 76:3089-3096.

Lee, S., Son, H., Lee, J., Min, K., Choi, K. J., Kim, J.-C. and Lee, Y.-W. 2011a. Functional analyses of two acetyl coenzymes A synthetases in the ascomycete Gibberella zeae. Eukaryot. Cell 10:1043-1052.

Lee, S., Son, H., Lee, J., Lee, Y.-R. and Lee, Y.-W. 2011b. A putative $\mathrm{ABC}$ transporter gene, $Z R A 1$, is required for zearalenone production in Gibberella zeae. Curr. Genet. 57:343-351.

Lee, S.-H., Lee, J., Lee, S., Park, E.-H., Kim, K.-W., Kim, M.-D., Yun, S.-H. and Lee, Y.-W. 2009. GzSNF1 is required for nor- mal sexual and asexual development in the ascomycete Gibberella zeae. Eukaryot. Cell 8:116-127.

Lee, S.-H., Lee, J., Nam, Y.J., Lee, S., Ryu, J.-G. and Lee, T. 2010c. Population structure of Fusarium graminearum from maize and rice in 2009 in Korea. Plant Pathol. J. 26:321-327.

Leslie, J. F. and Summerell, B. A. 2006. The Fusarium laboratory manual. Blackwell Professional, Ames, IA.

Lysøe, E., Klemsdal, S. S., Bone, K. R., Frandsen, R..J. N., Johansen, T., Thrane, U. and Giese, H. 2006. The PKS4 gene of Fusarium graminearum is essential for zearalenone production. Appl. Environ. Microbiol. 72:3924-3932.

Min, K., Lee, J., Kim, J.-C., Kim, S. G., Kim, Y.-H., Vogel, S., Trail, F. and Lee, Y.-W. 2010. A novel gene, ROA, is required for normal morphogenesis and discharge of ascospores in Gibberella zeae. Eukaryot. Cell 9:1495-1503.

Nussey, S. and Whitehead, S. 2001. Endocrinology: an integrated approach. Oxford: BIOS Scientific Bub., London, UK.

Sambrook, J. S. and Russell, D. 2001. Molecular cloning: A laboratory manual, Third ed. Cold Spring Harbor Laboratory Press, Cold Spring Harbor, NY.

Seong, K., Hou, Z., Tracy, M., Kistler, H. C. and Xu, J.-R. 2005. Random insertional mutagenesis identifies genes associated with virulence in the wheat scab fungus Fusarium graminearum. Phytopathology 95:744-750.

Smeds, A. I. Eklund, P. C., Sjoholm, R. E., Willfor, S. M., Nishibe, S., Deyama, T. and Holmbom, B. R. 2007. Qunatification of a broad spectrum of lignans in cereals, oilseeds, and nuts. $J$. Agric. Food Chem. 55:1337-1346.

Son, H., Lee, J., Park, A. R. and Lee, Y.-W. 2011. ATP citrate lase is required for normal sexual and asexal development in Gibberella zeae. Fungal Genet. Biol. 48:408-417.

Wang, L. Q. 2002. Mammalian phytoestrogens: enterodiol and enterolactone. J. Chromatogr. B Analyt. Technol. Biomed. Life Sci. 777:289-309.

Wolf, J. C. and Mirocha, C. J. 1973. Regulation of sexual reproduction in Gibberella zeae (Fusarium roseum 'Graminearum'). Can. J. Microbiol. 19:725-734.

Yu, J.-H., Hamari, Z., Han, K.-H., Seo, J.-A., Reyes-Dominguez, Y. and Scazzocchio, C. 2004. Double-Joint PCR: A PCRbased molecular tool for gene manipulations in filamentous fungi. Fungal Genet. Biol. 41:973-981. 